\title{
Evidências adicionais de validade da UWES-9 em amostras brasileiras
}

Maria Cristina Ferreira. Universidade Salgado de Oliveira.

Felipe Valentini. Universidade Salgado de Oliveira.

Bruno Figueiredo Damásio. Universidade Federal do Rio de Janeiro.

Luciana Mourão. Universidade Salgado de Oliveira.

Juliana Barreiros Porto. Universidade de Brasília.

Renata Silva de Carvalho Chinelato. Centro Universitário Estácio Juiz de Fora.

Vladimir Pinto Novaes. Universidade Salgado de Oliveira.

Michelle Morelo Pereira. Universidade Federal Fluminense.

\section{Resumo}

O engajamento no trabalho consiste em um estado motivacional positivo manifesto em sentimentos de vigor, absorção e dedicação ao trabalho. Para avaliá-lo, foi desenvolvida a Escala de Engajamento no Trabalho de Utrecht. O presente trabalho reuniu evidências de validade da versão reduzida da escala (UWES-9) por meio de análises de estrutura interna, de invariância por gênero e setor de trabalho (público ou privado), e de relações com variáveis externas. A amostra foi composta por 2.897 trabalhadores de ambos os sexos (57,3\% do sexo feminino), com média de idade de 32,8. Os modelos indicaram que a estrutura unidimensional obteve melhor ajuste e que os parâmetros dos itens não foram significativamente enviesados pelo sexo, setor e tempo de trabalho. A escala apresentou correlações positivas fortes com sentimentos positivos associados ao trabalho. As propriedades psicométricas do instrumento recomendam seu uso futuro em investigações destinadas a avaliar o engajamento no trabalho em amostras brasileiras.

Palavras-chave: comportamento organizacional; psicologia positiva; validade estatística.

\begin{abstract}
Additional validity evidences of UWES-9 in Brazilian samples. Work engagement is a positive motivational state which is expressed in vigor, absorption and dedication feelings toward work. The Utrecht Work Engagement Scale was developed to measure this phenomenon. The present work collected validity evidence for the reduced scale (UWES-9) concerning its internal structure, gender and work sector (public and private) invariance and relationships with external variables. The sample consisted of 2,897 employees from both genders (57.3\% were female), mean age equal to 32.8. The models indicated a unidimensional structure fit to the data, and the items parameters were not significantly biased due to gender, work sector, and job tenure. The scale showed positive strong correlations with positive feelings toward work. The psychometric properties of the scale recommend its future adoption in researchers about work engagement in Brazilian samples.
\end{abstract}

Keywords: organizational behavior; positive psychology; statistical validity. 


\section{Resumen}

Evidencias adicionales de validez de la UWES-9 en muestras brasileñas. El engagement en el trabajo es un estado de motivación positiva expresado en sentimientos de vigor, absorción y dedicación al trabajo. La Escala de Engagement en el Trabajo fue desarrollada para evaluar este constructo. El presente trabajo presentó evidencias de validez de la escala reducida (UWES-9) acerca de su estructura interna, invariancia de género y sector del trabajo (público y privado) y relaciones con variables externas. La muestra fue compuesta por 2.897 trabajadores (57,3\% mujeres), con una edad media de 32,8 años. Los modelos indicaron que la estructura de una dimensión se ajustó a los datos, y los parámetros de los ítems no fueron significativamente influenciados por las variables género, sector de trabajo y tiempo de trabajo. La escala mostró correlaciones positivas fuertes con sentimientos positivos acerca del trabajo. Las propiedades psicométricas de la escala recomiendan su uso futuro en investigaciones acerca del engagement en el trabajo en muestras brasileñas.

Palabras clave: conducta organizacional; psicología positiva; validación estadística.

Desde o surgimento da Psicologia Positiva (Seligman \& Csikszentmihalyi, 2000), há um número crescente de investigações dedicadas à análise dos antecedentes de resultados positivos em ambientes organizacionais. O engajamento no trabalho, que se caracteriza como um estado psicológico positivo relacionado ao trabalho, de caráter motivacional e se reflete no desejo de realmente contribuir para o sucesso organizacional (Schaufeli, 2013), é um dos construtos positivos que tem despertado o interesse desses estudiosos.

Schaufeli, Salanova, González-Romá e Bakker (2002) caracterizam o engajamento no trabalho como um estado psicológico difuso, de natureza afetivo-cognitiva, que se manifesta na dedicação, vigor e absorção ao trabalho. O vigor associa-se a níveis elevados de energia, que levam os empregados a vivenciarem seu trabalho como algo estimulante, que justifica envidar esforços e persistir na presença de dificuldades. A dedicação, por sua vez, concretiza-se no envolvimento com o próprio trabalho e nos sentimentos de que ele é algo significativo e que desperta entusiasmo e orgulho. A absorção, por fim, traduz-se em uma elevada concentração, que faz com que os empregados não percebam o tempo passar e não consigam desligar-se do trabalho.

Fundamentando-se no modelo tridimensional de engajamento no trabalho, Schaufeli et al. (2002) desenvolveram a Escala de Engajamento de Utrecht (UWES-17), inicialmente composta por 24 itens e que, após procedimentos de refinamento, ficou composta por 17 itens. Estudos posteriores, conduzidos em amostras de diferentes países, com trabalhadores pertencentes a diversos grupos ocupacionais, confirmaram que a escala apresentava propriedades psicométricas adequadas (Fong \& Ng, 2012; Nerstad, Richardsen, \& Martinussen, 2010; Vazquez, Magnan, Pacico, Hutz, \& Schaufeli, 2015).
Os resultados favoráveis obtidos nas pesquisas com a UWES-17 levaram Schaufeli, Bakker e Salanova (2006) a proporem uma versão reduzida da escala, denominada de UWES-9, composta por nove itens, distribuídos igualmente entre os três fatores originais da escala. Adotando procedimentos de análise fatorial confirmatória, estudos têm encontrado evidências que apoiam tanto um modelo de estrutura da UWES-9 em três fatores, como modelos unifatoriais. A estrutura tri-fatorial da UWES-9 obteve índices melhores de ajuste que a estrutura unifatorial em pesquisas realizadas com amostras européias (Nerstad et al., 2010) e asiáticas (Panthee, Shimazu, \& Kawakami, 2014). Contudo, outros estudos, conduzidos em amostras asiáticas (Fong \& Ho, 2015; Shimazu et al., 2008) e africanas (Bruin \& Henn, 2013), bem como o estudo transcultural de Klassen et al. (2012), com professores da Austrália, Canadá, China (Hong-Kong), Indonésia e Oman, constataram que a estrutura unifatorial da UWES-9 apresentava melhores índices de ajuste e de consistência interna, quando comparados à estrutura de três fatores. A investigação de Wefald, Mills, Smith e Downey (2012), nos Estados Unidos, por sua vez, concluiu que nem a estrutura tri-fatorial e nem tampouco a unifatorial produziram bons índices de ajuste aos dados.

No Brasil, o estudo de validação recentemente conduzido por Vazquez et al. (2015) também observou que a UWES-9 adequava-se mais a uma estrutura unifatorial. No entanto, os próprios autores sugerem a necessidade de novos estudos que contribuam para aprofundar o debate acerca de a escala ser melhor descrita por uma estrutura uni ou tri-fatorial. Justificam-se, assim, estudos adicionais sobre a UWES-9 em amostras brasileiras, que possam contribuir com novas evidências acerca da estrutura mais adequada à descrição da escala 
e acerca de sua validade convergente. Desse modo será possível disponibilizar aos estudiosos das organizações brasileiras um instrumento para a avaliação do engajamento no trabalho que seja curto e que apresente boas características psicométricas. A relevância desse esforço reside no fato de o referido construto vir se revelando um bom preditor de diversas atitudes e comportamentos positivos no trabalho (Schaufeli, 2013). Fundamentando-se em tais considerações, o presente trabalho objetivou reunir evidências adicionais de validade de estrutura interna e de invariância por gênero e setor de trabalho (público ou privado), bem como da possível influência do tempo de trabalho, além de relações com variáveis externas (validade convergente), da Escala de Engajamento no Trabalho-Versão Reduzida (UWES-9), em amostras brasileiras. Ademais, buscou-se avaliar a consistência interna dos escores estimados por meio da versão reduzida da UWES-9.

Estudos com a escala têm demonstrado que parece não haver diferenças de gênero para o engajamento no trabalho, mas diferenças entre tipos de trabalho e idade foram encontradas (Schaufeli, 2012). Assim, buscou-se testar se essas variáveis gerariam viéses na estimação dos escores latentes de engajamento. O setor de trabalho foi utilizado como um proxy para tipos de trabalho. Tempo de serviço foi usado por se sobrepor à idade, uma vez que quanto maior o tempo de serviço, maior tende a ser a idade.

No que se refere à variáveis externas, Schaufeli e Salanova (2008) revisaram a literatura e apontam para a relação entre engajamento e neuroticismo, sendo que funcionários engajados apresentam baixo neuroticismo; e entre engajamento e autoeficária, sendo esta relação positiva. A revisão também aponta para os seguintes consequentes do engajamento: maior satisfação com o trabalho e comprometimento organizacional, menor intenção de deixar a organização, boa saúde mental, aumento dos comportamentos extrapapéis e melhor desempenho no trabalho.

A partir dos resultados de estudos anteriores, foram selecionadas as seguintes variáveis para teste de validade convergente: neuroticismo, desempenho de papéis, avaliações autorrefentes, florescimento no trabalho, satisfação com a vida e com o trabalho e paixão pelo trabalho. Hipotetizou-se que a UWES-9 apresentaria correlações positivas fortes com atitudes e comportamentos positivos (avaliações autorreferentes, satisfação com a vida, satisfação no trabalho, florescimento no trabalho, paixão harmoniosa pelo trabalho, desempenho no trabalho) e correlação negativa moderada ou fraca com um traços negativo não diretamente relacionado ao trabalho (neuroticismo).

\section{Método}

\section{Participantes}

A amostra foi composta a partir de bancos de dados de cinco diferentes pesquisas brasileiras, o que resultou em um total de 2.897 participantes, provenientes principalmente dos estados do Rio de Janeiro $(n=$ 1.429), Minas Gerais $(n=964)$ e Mato Grosso $(n=504)$. Suas idades variaram de 18 a 79 anos (Média $=32,8$ anos; $D P=10,6$; 39 participantes não informaram este dado), sendo a maior parte do sexo feminino (57,3\%; 12 participantes não informaram este dado). A maioria dos participantes afirmou ter ensino superior completo ou incompleto (77,3\%; 28 participantes não informaram este dado).

\section{Instrumentos}

Todos os participantes $(n=2.897$ ) responderam à versão reduzida da Escala Utrecht de Engajamento no Trabalho (UWES-9; Schaufeli et al., 2006). Essa medida é composta por nove itens, que descrevem possíveis sentimentos de vigor, dedicação e absorção ao trabalho, para os quais o participante deve indicar a frequência com que tais sentimentos ocorrem. Exemplo de item: 'Em meu trabalho, sinto-me repleto (cheio) de energia'. Os itens são respondidos em uma escala do tipo Likert de sete pontos (variando de 'nunca' a 'sempre').

Os demais instrumentos foram respondidos por somente parte da amostra total. As avaliações autorreferentes $(n=829)$ foram mensuradas por meio da versão brasileira da Escala de Avaliações Autorreferentes (Ferreira et al., 2013), adaptada a partir da escala desenvolvida por Judge, Erez, Bono e Thoresen (2003). O instrumento é composto de 12 itens, a serem respondidos em uma escala Likert de cinco pontos. Exemplo de item: 'Tenho confiança de que obterei o sucesso que mereço na vida'. O Alfa de Cronbach foi igual a 0,79 no atual estudo.

A Escala de Desempenho de Papéis de Trabalho (Williams \& Anderson, 1991) foi adotada na avaliação do desempenho no trabalho $(n=946)$. Ela possui sete itens em formato de escala Likert de cinco pontos. Exemplo de item: 'Executo as tarefas que são de mim esperadas'. O Alfa de Cronbach foi igual a 0,71 no presente estudo. 
Para mensurar o neuroticismo $(n=946)$ foi utilizada uma das Escalas do Inventário dos Cinco Grandes Fatores de Personalidade (John, Donahue, \& Kentle, 1991), adaptado para amostras brasileiras por Andrade (2008). A subescala de neuroticismo contém seis itens, respondidos em uma escala tipo Likert de cinco pontos. Exemplo de item: 'Vejo-me como alguém que fica nervoso facilmente'. O Alfa de Cronbach foi igual a 0,77 no presente estudo.

O florescimento no trabalho $(n=464)$ foi mensurado pela Escala de Florescimento no Trabalho (Diener et al., 2010), adaptada ao contexto do trabalho por Mendonça, Caetano, Ferreira, Sousa e Silva (2014). O instrumento é composto de oito itens, respondidos em uma escala Likert de sete pontos. Exemplo de item: 'Em meu trabalho, as pessoas me respeitam'. O Alfa de Cronbach foi igual a 0,83 no presente estudo.

Para avaliação da satisfação com a vida $(n=1.047)$, adotou-se a escala proposta por Diener, Emmons, Larsen e Griffin (1985) e adaptada ao contexto brasileiro por Gouveia, Barbosa, Andrade e Carneiro (2005). A escala compõe-se de cinco itens respondidos em escalas tipo Likert de sete pontos. Exemplo de item: 'Estou satisfeito com minha vida'. O Alfa de Cronbach foi igual a 0,87 no presente estudo.

A satisfação no trabalho $(n=829)$ foi mensurada por meio da Escala de Satisfação Geral no Trabalho de Silva e Ferreira (2009). O instrumento é composto por cinco itens, respondidos por meio de uma escala Likert de cinco pontos. Exemplo de item: 'Sinto-me satisfeito com meu trabalho atual'. O Alfa de Cronbach foi igual a 0,91 no presente estudo.

$\mathrm{Na}$ avaliação da paixão pelo trabalho $(n=504)$ adotou-se a Escala de Paixão pelo Trabalho de Vallerand e Houlford (2003), em versão brasileira adaptada por Pereira, Ferreira e Valentini (2016). Ela é composta por duas dimensões: paixão harmoniosa e paixão obsessiva, com sete itens cada, respondidos em escalas tipo Likert de sete pontos. Na presente pesquisa, porém, apenas a escala de paixão harmoniosa foi utilizada (Exemplo de item: 'Meu trabalho reflete as qualidades que eu gosto em mim'), tendo apresentado um Alfa de Cronbach de 0,90.

\section{Procedimentos de Coleta e Análise dos Dados}

Os dados das cinco pesquisas foram coletados on-line. Todos os participantes foram voluntários e assentiram a participação e concordância com o Termo de Consentimento Livre e Esclarecido (TCLE). Ademais, o projeto de pesquisa foi aprovado pelo comitê de ética da instituição de um dos autores (parecer 465.548).
Por meio de uma análise exploratória descritiva dos dados, verificou-se que os itens apresentavam restrições de variabilidade, bem como violação da normalidade multivariada (coeficiente de Mardia = 155; bicaudal, $p<0,001)$. Considerando esses resultados e ponderando o tamanho da amostra, para a estimação de parâmetros na modelagem por equações estruturais, optou-se pela utilização do método de estimação Robust Maximum Likelihood (MLR), robusto à violação do pressuposto da distribuição normal dos dados (Satorra \& Bentler, 2001). O ajuste dos modelos aos dados foi avaliado por meio dos seguintes indicadores e respectivos valores de referência: $\chi^{2} / \mathrm{gl}$ <; BentlerComparative Fit Index - CFI > 0,95; Tucker-Lewis Index - TLI > 0,95; Root Mean Square Error of Approximation - RMSEA $<0,05$; Standardized Root Mean Square Residual - SRMR < 0,09.

Considerando que a literatura referente à UWES-9 apresenta evidências para diferentes estruturas, na presente pesquisa foram testados modelos de um e três fatores. Para avaliar se a versão reduzida da UWES mantinha a mesma estrutura fatorial entre os participantes do sexo masculino $(n=1.229)$ e feminino $(n=1.651)$ e entre trabalhadores do setor público $(n=291)$ e privado $(n=$ 651), a invariância dos parâmetros dos itens foi testada por meio da análise fatorial confirmatória multigrupo (AFCMG). Para tanto, foram testados modelos nos quais foram fixados o número de itens e fatores (invariância configural), cargas fatoriais (invariância métrica) e interceptos (invariância escalar).

Ademais, buscou-se avaliar se o tempo de trabalho produzia viéses na estimação dos escores latentes de engajamento. Considerando que a variável tempo de trabalho é do tipo de razão, a invariância dos parâmetros foi testada por meio da modelagem Multiple Indicators and Multiple Causes - MIMIC (Muthén, 1988). Esse modelo prevê o teste de efeitos diretos e indiretos da variável tempo de trabalho sobre os itens. Efeitos diretos do tempo de trabalho sobre os itens indicam que essa variável impacta na parametrização dos itens. Contudo, na modelagem por equações estruturais, o modelo com todos os efeitos diretos e indiretos do tempo de trabalho sobre os itens é indeterminado. Para resolver esse problema, estimou-se primeiramente um modelo apenas com efeitos indiretos (modelo sem viés) e buscou-se avaliar os índices de modificação relacionados aos efeitos diretos do tempo de trabalho sobre os itens (modelo com viés). Finalmente, as relações entre o engajamento e as variáveis externas foram calculadas por meio de correlações de Pearson, com escores fatoriais das escalas estimados por meio da modelagem por equações estruturais. 


\section{Resultados}

\section{Análise da Estrutura Interna da UWES-9}

Inicialmente, testou-se a estruturação da UWES-9 em uma ou três dimensões. Os indicadores de ajuste desses modelos são apresentados na Tabela 1.

Os dois primeiros modelos testados apresentaram indicadores de ajuste aquém do esperado. Assim, foram testados dois modelos alternativos (um unifatorial e outro de três fatores correlacionados), sendo que, em ambos, foram estimados livremente correlações de erros entre quatro pares de itens, a saber: 1-2, 3-4, 5-6 e 7-8. As correlações residuais entre esses itens foram adotadas devido aos elevados valores dos índices de modificação obtidos nas análises iniciais.

O modelo reespecificado com três fatores correlacionados (Modelo 4, Tabela 1) apresentou ajuste aos dados levemente superior ao modelo unidimensional reespecificado. Ressalta-se que as diferenças de TLI, CFI, RMSEA e SRMR entre esses dois modelos são praticamente desprezíveis e indicam que, considerando apenas os indicadores de ajuste, não é possível concluir pelo melhor modelo estrutural. Ademais, as cargas fatoriais e erros-padrão de estimativa (valores não apresentados nas tabelas) são semelhantes entre os dois modelos, o que também torna os resultados inconclusivos.

Contudo, as correlações entre as três dimensões do Modelo 4 são muito altas $(r \geq 0,96)$. Além disso, os valores calculados para a Variância Média Extraída (VME) ficaram, para o Modelo 4, entre 0,49 e 0,72, sendo inferiores aos coeficientes de determinação para a relação entre os fatores ( $r^{2}$ entre 0,91 e 0,97$)$. Tais resultados indicam a ausência de evidência de validade discriminante entre as dimensões latentes. Em outras palavras, ainda que o modelo de três fatores tenha se ajustado bem aos dados, não há evidência de que o escore de uma dimensão latente seja suficientemente distinto dos escores das outras duas dimensões. Considerando essas limitações, adotou-se o modelo unidimensional como o melhor modelo para a estrutura da versão brasileira da UWES (as cargas fatoriais são apresentadas na Tabela 3, última coluna - 'Modelo Original').

\section{Análise de Invariância da UWES-9}

Considerado o modelo de estrutura unidimensional, buscou-se, por meio da análise fatorial multigrupo (AFCMG), avaliar a invariância dos parâmetros dos itens entre os participantes do gênero masculino e feminino, bem como entre trabalhadores do setor público e setor privado. Os resultados da AFCMG são indicados na Tabela 2. As desprezíveis reduções no valor de CFI (CFI <0,01, entre todas as comparações) indicaram que o modelo, as cargas fatoriais e os interceptos foram invariantes entre os gêneros, bem como entre os trabalhadores dos setores público e privado.

Além da análise multigrupo, buscou-se avaliar a influência do tempo de trabalho sobre a estimação dos parâmetros dos itens da UWES. Na modelagem Multiple Indicators Multiple Causes (MIMIC), o primeiro modelo estimado (modelo sem viés) ajustou-se bem aos dados (Tabela 3). No entanto, os índices de modificação do modelo sem viés apontaram para alterações significativas no ajuste do modelo, caso fossem estimados livremente os parâmetros de regressão diretos entre o tempo de trabalho e os itens 3, 4, 5 e 6. Assim, esses coeficientes de regressão foram estimados livremente no segundo modelo (modelo com viés, Tabela 3 ). Tais resultados indicam possíveis vieses do tempo de trabalho sobre as respostas aos itens 3, 4, 5 e 6. Nesse sentido, os indicadores de ajuste do modelo com viés são levemente superiores ao modelo sem viés $(\Delta \mathrm{CFI}<0,01)$. No modelo com viés, os coeficientes de regressão diretos entre tempo de trabalho e os itens 3, 4, 5 e 6 apresentaram

Tabela 1. Indicadores de Ajuste da Modelagem por Equações Estruturais.

\begin{tabular}{|c|c|c|c|c|c|c|}
\hline Modelo & $\chi^{2}(\mathrm{gl})$ & TLI & CFI & RMSEA (IC 90\%) & SRMR & $\mathrm{BIC}$ \\
\hline 1. Um Fator Geral & $1448,44(27)$ & 0,834 & 0,875 & $\begin{array}{c}0,135 \\
(0,129-0,141)\end{array}$ & 0,043 & 80393,59 \\
\hline $\begin{array}{l}\text { 2. Três Fatores Correlacionados } \\
\text { ( } r \text { entre } 0,85 \text { e } 0,96)\end{array}$ & $1081,59(24)$ & 0,861 & 0,907 & $\begin{array}{c}0,123 \\
(0,117-0,130)\end{array}$ & 0,050 & 79653,24 \\
\hline 3. Um Fator Geral (reespecificado*) & $217,01(23)$ & 0,973 & 0,983 & $\begin{array}{c}0,054 \\
(0,048-0,061)\end{array}$ & 0,020 & 78280,47 \\
\hline $\begin{array}{l}\text { 4. Três Fatores (reespecificado) } \\
\text { ( } r \text { entre } 0,96 \text { e } 0,98)\end{array}$ & $185,90(20)$ & 0,974 & 0,985 & $\begin{array}{c}0,054 \\
(0,048-0,061)\end{array}$ & 0,017 & 78253,63 \\
\hline
\end{tabular}

* reespecificado = livre estimação das correlações entre os erros dos seguintes itens: 1 e 2, 3 e 4, 5 e 6, 7 e 8; $\chi^{2}$ : qui-quadrado; gl: graus de liberdade; TLI: Tucker Lewis Index; CFI: ComparativeFix Index; RMSEA: Root Mean Square ErrorofApproximation; SRMR: Standardized Root Mean Square Residual. 
Tabela 2. Análise Fatorial Confirmatória Multigrupo (AFCMG) para as Variáveis Gênero e Setor.

\begin{tabular}{|c|c|c|c|c|c|c|}
\hline $\begin{array}{l}\text { Gênero } \\
\text { (homens x mulheres) }\end{array}$ & $\chi^{2}(\mathrm{gl})$ & CFI & TLI & RMSEA & AIC & $\mathrm{BIC}$ \\
\hline Configural & $245,66(46)$ & 0,983 & 0,973 & 0,055 & 77738,01 & 78107,88 \\
\hline Métrica & $267,60(54)$ & 0,981 & 0,975 & 0,052 & 77737,41 & 78059,54 \\
\hline Escalar & $294,35(62)$ & 0,980 & 0,977 & 0,051 & 77742,88 & 78017,29 \\
\hline $\begin{array}{l}\text { Setor } \\
\text { (público x privado) }\end{array}$ & $\chi^{2}(\mathrm{gl})$ & CFI & TLI & RMSEA & AIC & $\mathrm{BIC}$ \\
\hline Configural & $168,50(46)$ & 0,969 & 0,952 & 0,075 & 25047,74 & 25151,47 \\
\hline Métrica & $182,19(54)$ & 0,968 & 0,957 & 0,071 & 25041,21 & 25131,56 \\
\hline Escalar & $192,76(62)$ & 0,967 & 0,962 & 0,067 & 25029,46 & 25106,43 \\
\hline
\end{tabular}

$\mathrm{X}^{2}$ : qui-quadrado; gl: graus de liberdade; TLI: Tucker Lewis Index; CFI: Comparative Fix Index; RMSEA: Root Mean Square Error of Approximation; AIC: Akaike Information Criterion; BIC: Bayesian Information Criterion; Configural: estrutura fatorial fixa entre os grupos; Métrica: estrutura fatorial e cargas fatoriais fixas entre os grupos; Escalar: estrutura fatorial, cargas fatoriais e interceptos fixos entre os grupos.

tamanhos de efeito muito baixos. Além disso, ao comparar os modelos com e sem viés, percebe-se que as diferenças das cargas fatoriais (coeficientes de regressão entre a variável latente engajamento e os itens) também são pequenas $(\Delta \beta \leq 0,01)$.

Para ampliar a discussão sobre o efeito de viés do tempo de trabalho sobre a escala de engajamento, comparou-se os escores latentes, de todos os participantes, estimados pelos modelos com e sem viés. Tais diferenças variaram de 0 a 0,062 (média $=-0,013$ ), numa indicação de que o modelo sem viés subestima, em média, os escores latentes em 0,013 pontos. As maiores diferenças entre os escores latentes foram observadas para os participantes com mais de 30 anos de trabalho (média da diferença $=0,03$ ).

\section{Análise de Consistência Interna da UWES-9}

Para o modelo unidimensional, o valor do alfa de Cronbach foi igual a 0,93 e o da Confiabilidade Composta (CC) igual a 0,91. Também se estimou a precisão dos escores por meio da Variância Média Extraída (VME), cujo resultado foi igual a 0,59. Considerando que as cargas fatoriais foram razoavelmente homogêneas (DP de cargas $=0,09$ ), a VME e a CC indicam que a maior parte da variância dos itens pode ser explicada, de maneira precisa, pelo fator latente Engajamento.

Tabela 3. Coeficientes de Regressão Padronizados dos Modelos com e sem Viés da Variável Tempo de Trabalho.

\begin{tabular}{|c|c|c|c|c|c|}
\hline \multirow[b]{2}{*}{ Itens } & \multicolumn{2}{|c|}{$\begin{array}{c}\text { Modelo com viés } \\
\text { (efeitos diretos e indiretos) }\end{array}$} & \multicolumn{2}{|c|}{$\begin{array}{l}\text { Modelo sem viés } \\
\text { (efeitos indiretos) a }\end{array}$} & \multirow{2}{*}{$\begin{array}{c}\text { Modelo original } \\
\text { (sem TT) } \\
\text { Engaj }\end{array}$} \\
\hline & Engaj & TT & Engaj & TT & \\
\hline engaj2 & 0,822 & n.s. & 0,823 & - & 0,823 \\
\hline engaj3 & 0,877 & $-0,043$ & 0,868 & - & 0,870 \\
\hline engaj6 & 0,687 & 0,042 & 0,695 & - & 0,694 \\
\hline engaj7 & 0,772 & n.s. & 0,772 & - & 0,772 \\
\hline engaj8 & 0,788 & n.s. & 0,789 & - & 0,788 \\
\hline engaj9 & 0,561 & n.s. & 0,563 & - & 0,562 \\
\hline $\mathrm{BIC}$ & 68973,44 & & 68999,36 & & não-aninhado ${ }^{b}$ \\
\hline $\mathrm{CFI}$ & 0,984 & & 0,979 & & não-aninhado ${ }^{b}$ \\
\hline
\end{tabular}

a os efeitos diretos entre a variável externa (tempo de trabalho) e os itens foram fixados em 0 , para o modelo sem viés; ${ }^{\text {b }}$ por não considerar a variável tempo de trabalho esse modelo não é aninhado aos demais e, por esse motivo, não foram apresentados os indicadores de ajuste; Todos os parâmetros apresentados foram estatisticamente significativos, $p<0,01$, à exceção daqueles apresentados com "n.s." (não significativo); Engaj: engajamento; TT: Tempo de trabalho. 


\section{Relações entre os Escores de Engajamento e Variáveis Externas}

Observaram-se correlações fracas e moderadas entre engajamento e neuroticismo $(r=-0,24)$, desempenho no trabalho $(r=0,32)$ e satisfação com a vida $(r=0,39)$. Ademais, correlações fortes entre engajamento e florescimento no trabalho $(r=0,74)$, satisfação com o trabalho $(r=0,77)$, avaliações autorreferentes $(r=0,52)$ e paixão pelo trabalho $(r=0,78)$ foram obtidas.

\section{Discussão}

Foi objetivo do presente trabalho reunir evidências adicionais de validade de estrutura interna e de invariância por gênero e setor de trabalho (público ou privado), bem como da possível influência do tempo de trabalho, além de relações com variáveis externas (validade convergente), da Escala de Engajamento no Trabalho-Versão Reduzida (UWES-9), em amostras brasileiras.

A versão reduzida da UWES apresenta a vantagem de ser mais parcimoniosa e de fácil aplicação, sem perda de confiabilidade dos escores. Além disso, no campo da psicologia, o interesse no desenvolvimento de escalas curtas tem crescido consideravelmente (Schweizer, 2011), mesmo para as medições de traços, tipicamente realizadas com escalas mais longas (Romero, Villar, Gómez-Fraguela, \& López-Romero, 2012). No caso específico da pesquisa na área organizacional, as escalas mais curtas mostram-se necessárias porque geralmente integram instrumentos mais longos, destinados à avaliação de uma série de variáveis incluídas em um modelo de pesquisa. No entanto, quanto maior a extensão do instrumento, maior o risco de fadiga e tédio para os participantes, o que pode comprometer a qualidade dos dados.

$\mathrm{Na}$ avaliação da estrutura interna, foram testados os modelos de um e três fatores, sendo que ambos apresentaram, inicialmente, índices de ajuste abaixo do recomendado. Por outro lado, os índices de modificação para ambos os modelos sugeriram que a inserção de covariância residual entre os itens 1-2, 3-4, 5-6 e 7-8 melhoraria significativamente $o$ ajuste dos modelos.

A inserção de covariância residual entre indicadores é um ponto crítico na literatura sobre análise fatorial confirmatória. Pesquisadores têm salientado a importância de inserir estes tipos de covariâncias apenas quando há explicação teórica ou outras justificativas plausíveis (Hayduk, Cummings, Boadu, Pazderka-Robinson, \& Boulianne, 2007). No presente estudo, por um lado, os pares de itens 1-2 e 3-4 são propostos para avaliar a mesma dimensão teórica e possuem conteúdos semelhantes, por outro, o mesmo não acontece para os pares de itens 5-6 e 7-8, que deveriam avaliar as dimensões vigor, absorção, dedicação e absorção, respectivamente.

De todo modo, a necessidade de inserção dessas covariâncias atesta limitações da escala em separar adequadamente o construto engajamento no trabalho nas dimensões de vigor, dedicação e absorção. Essa interpretação é corroborada nas análises subsequentes, nas quais evidenciou-se que as três dimensões latentes apresentaram covariâncias demasiadamente altas entre si, o que impede a existência de validade discriminante entre os construtos. Considerando-se, portanto, todas as limitações da estrutura de três fatores, optou-se pela utilização da estrutura unidimensional como a mais parcimoniosa para a descrição da UWES-9 na presente amostra, e por sua utilização nas análises subsequentes, o que se mostra congruente com diferentes investigações que também concluíram que a UWES-9 era melhor descrita por uma estrutura unifatorial (Bruin \& Henn, 2013; Fong \& Ho, 2015; Klassen et al., 2012; Shimazu et al., 2008; Vazquez et al., 2015).

Importante esclarecer que, em termos práticos, a solução unifatorial implica que o pesquisador ou o profissional que for utilizar a medida deve considerar o engajamento como um todo, ou seja, não deve avaliar de forma específica as dimensões vigor, absorção e dedicação, mas sim considerar que tais elementos compõem o engajamento no trabalho. Qualquer análise de resultado ou conclusão de pesquisa em amostras brasileiras, advinda desta solução unifatorial e reduzida da escala UWES-9, deve ater-se ao engajamento como variável latente única, podendo a mesma ser relacionada a outros construtos e variáveis organizacionais ou pessoais, a depender do desenho e dos interesses de pesquisa.

Os resultados das análises fatoriais confirmatórias multigrupo (AFCMG) demonstraram que a escala não apresentou vieses de resposta para homens e mulheres e nem tampouco para trabalhadores do setor público e do setor privado. Nesse sentido, pode-se afirmar que os escores de engajamento no trabalho obtidos por meio da escala são invariantes para gênero e setor de trabalho e que esses grupos podem ser comparados entre si (Sass, 2011). Tais evidências dão, assim, sustentação aos estudos que vêm evidenciando a não existência de diferenças significativas entre os gêneros (Fong \& Ng, 2012; Vasquez et al., 2015, Schaufeli, 2012) e entre trabalhadores do setor público e privado (Vigoda-Gadot, Eldor, \& Schohat, 
2012) no que diz respeito ao engajamento no trabalho. No presente estudo, essas diferenças foram testadas de forma mais rigorosa. Entretanto, é importante que estudos futuros comparem a estrutura para tipos de trabalho considerando o grau de complexidade e autonomia do trabalho.

Vale registrar que o estudo de Vasquez et al., 2015 apontou validade de conteúdo e validade de construto tanto para os 17 itens da escala, quanto para os nove itens da versão reduzida, apontando diferenças de engajamento em relação à idade. O presente estudo avança em relação aos resultados já obtidos por Vasquez et al. (2015), por apresentar outros indicadores de validade a partir da invariância por gênero, setor (público ou privado) e tempo de trabalho. Nesse sentido, aumenta-se a possibilidade de uso da escala para diferentes públicos e contextos, uma vez que foi encontrada invariância para gênero e setor de trabalho.

O tempo de trabalho, entretanto, tende a influenciar as respostas ao instrumento. Os resultados da análise MIMIC indicaram que os itens 3, 4, 5 e 6 tendem a subestimar, levemente, os escores de engajamento, conforme o aumento do tempo de trabalho dos participantes. Contudo, a diferença nos escores latentes estimados pelos modelos com e sem viés foi pequena. Considerando que o erro padrão dos escores latentes estimados por meio do modelo sem viés foi igual a 0,32, a média da diferença dos escores com e sem viés (igual a 0,01) corresponde a apenas $4 \%$ do erro padrão (e o valor máximo da diferença corresponde a $19,5 \%$ do erro padrão). Nesse sentido, ainda que um modelo que desconsidere o tempo de trabalho possa subestimar os escores latentes do engajamento, tal subestimação é muito pequena e, para quase a totalidade dos casos, praticamente desprezível. Esses resultados estão em sintonia com o estudo citado por Schaufeli (2012) que indica uma discreta diferença para a idade, sendo os funcionários mais velhos um pouco mais engajados que os novos.

No que se refere à precisão dos escores, os indicadores de consistência foram altos. Surpreendeu, um pouco, o valor de confiabilidade composta (CC) levemente inferior ao alfa de Cronbach. Ainda que a Confiabilidade Composta tenda a apresentar valores superiores ao alfa, a livre estimação de covariâncias residuais tende a diminuir a CC. Essa tendência pode explicar o valor da CC levemente abaixo do alfa, no presente estudo. De todo modo, os dados ora obtidos mostram-se consistentes com os estudos (Klassen et al., 2012) que também têm apresentado índices de consistência interna elevados, especialmente quando a escala é considerada unifatorial.

Em relação à correlação dos escores da UWES-9 com variáveis externas, verificou-se que a escala de engajamento no trabalho apresentou correlações fortes e positivas com os escores de florescimento no trabalho, de satisfação no trabalho e de paixão harmoniosa pelo trabalho. Tais achados confirmam os resultados anteriores obtidos pelos diversos autores que também vêm obtendo evidências de que o engajamento no trabalho se associa positivamente a atitudes positivas direcionadas ao trabalho (Klassen et al., 2012; Shimazu et al., 2008).

De acordo com Schaufeli (2013), o engajamento no trabalho caracteriza-se como um estado psicológico de natureza afetivo-cognitiva. Considerando-se que a satisfação no trabalho (vínculo afetivo com o trabalho decorrente de experiências laborais prazerosas; Siqueira, 2008), o florescimento no trabalho (sentimentos de prosperidade no e pelo trabalho expressos em emoções e motivações positivas dirigidas às atividades laborais) (Bono, Davies, \& Rasch, 2011) e a paixão harmoniosa pelo trabalho (sentimentos positivos em relação ao trabalho, que levam o indivíduo a investir força e energia interna na realização de suas tarefas laborais) (Vallerand \& Houlford, 2003) também consistem em expressões de sentimentos e afetos positivos focalizados no trabalho (Wefald et al., 2012), foi hipotetizado que todos esses construtos se apresentariam fortemente correlacionados, o que de fato ocorreu.

A correlação entre as avaliações autorreferentes e o engajamento no trabalho foi também elevada e positiva, conforme hipotetizado, embora inferior aos efeitos das relações entre engajamento, florescimento, satisfação no trabalho e paixão pelo trabalho harmoniosa. Tais resultados podem ser vistos como evidências de que as pessoas com escores mais elevados em avaliações autorreferentes tendem a ter uma visão mais positiva de si próprias e a avaliar seu trabalho de forma mais positiva (Bono et al., 2011), o que poderia estar levando-as a desenvolver também maior afetividade em relação ao trabalho. Cumpre ressaltar, porém, que o tamanho do efeito da relação entre engajamento e avaliações autorreferentes foi inferior aos efeitos das relações entre engajamento, florescimento, satisfação no trabalho e paixão harmoniosa pelo trabalho, numa indicação de que a tendência de se autoavaliar positivamente não interferiu nas correlações das outras três escalas com o engajamento no trabalho. 
A correlação do engajamento com o desempenho foi moderada, conforme previsto. Uma possível explicação para tal resultado é a de que o desempenho extrapapéis costuma sofrer maior impacto do engajamento do que o desempenho intra-papéis, que foi aqui avaliado, em virtude de a natureza energizadora do engajamento despertar muito mais a vontade de o indivíduo ir além de suas tarefas prescritas (como no caso do desempenho extrapapel) do que o simples desejo de ele apresentar um bom desempenho (Bakker, Demerouti, \& Sanz-Vegel, 2014). A satisfação com a vida também se correlacionou moderadamente com o engajamento no trabalho, e não fortemente, como hipotetizado. Tal resultado pode ser interpretado pelo fato de os focos desses dois construtos (vida e trabalho, respectivamente) serem distintos, muito embora ambos reflitam estados afetivos.

Verificou-se, ainda, que o engajamento no trabalho apresentou uma correlação negativa fraca com o neuroticismo, conforme hipotetizado, o que se mostra congruente com o resultado obtido recentemente por Zecca et al. (2015) e sinaliza que os indivíduos mais engajados em seu trabalho tendem a vivenciar menos emoções negativas. Em suma, esses resultados estão em sintonia com aqueles encontrados em estudos internacionais (Schaufeli \& Salanova, 2008) e reforçam as evidências de validade da escala.

Em relação às limitações da presente pesquisa, o fato de a amostra ter sido composta principalmente por participantes do Rio de Janeiro, Minas Gerais e Mato Grosso limita a generalização dos resultados para os demais estados brasileiros. Torna-se, assim, necessária a realização de novos estudos destinados a obter evidências adicionais de validade em outros contextos geográficos. Outrossim, todas as medidas adotadas no estudo foram de autorrelato e aplicadas conjuntamente, sem que tenha havido espaço temporal entre as mesmas, razão pela qual a variância comum ao método pode ter interferido nos resultados ora obtidos (Podsakoff, MacKenzie, \& Podsakoff, 2012). Outro ponto passível de crítica refere-se ao fato de que a ordem de aplicação da UWES-9 variou entre as amostras. Assim, fontes aleatórias e sistemáticas de erro podem ter impactado na estimação dos índices de ajuste da escala e no traço latente dos participantes. Entretanto, essa hipótese é pouco provável, haja vista que a escala apresentou adequados índices de ajuste para diferentes subgrupos.

Os achados deste estudo, aliados aos anteriormente descritos por Vasquez et al. (2015), atestam que a versão reduzida da Escala de Engajamento no Trabalho de
Utrecht (UWES-9) vem apresentando boas propriedades psicométricas em amostras brasileiras. Nesse sentido, ela pode ser adotada, por exemplo, em estudos futuros sobre a validade discriminante do engajamento no trabaIho, mediante a comparação do percentual de variância que o engajamento, a satisfação, a paixão harmoniosa e o florescimento no trabalho explicam, na predição de distintas atitudes e comportamentos positivos dirigidos ao trabalho. Ademais, os resultados da presente pesquisa apontam que a escala permite avaliar participantes com diferentes tempos de trabalho, embora os atuais achados sinalizem que seria mais interessante o controle dessa variável nas pesquisas futuras sobre o engajamento no trabalho.

Futuros estudos que busquem investigar a validade discriminante do engajamento no trabalho são pertinentes. Isto é, seria importante compreender em que medida o engajamento no trabalho se diferencia de outros construtos psicológicos positivos relacionados ao trabalho, tais como satisfação, flow, paixão harmoniosa e florescimento no trabalho. Ademais, são necessários mais estudos que busquem entender quais variáveis explicam o engajamento no trabalho, bem como o impacto do engajamento em distintas atitudes e comportamentos positivos dirigidos ao trabalho.

\section{Referências}

Andrade, J. M. (2008). Evidências de validade do Inventário dos Cinco Grandes Fatores de Personalidade para o Brasil (Tese de doutorado). Universidade de Brasília, Brasília, DF, Brasil. Recuperado de http:// repositorio.unb.br/handle/10482/1751

Bakker, A. B., Demerouti, E., \& Sanz-Vegel, A. I. (2014). Burnout and work engagement:The JD-R approach. Annual Review of Organizational Psychology and Organizational Behavior, 1, 389-411. doi: 10.1146/ annurev-orgpsych-031413-091235

Bono, J., Davies, S., \& Rasch, R. (2011). Some traits associated associated with flourishing at work. In K. Cameron \& G. Spreitzer (Orgs.), The Oxford handbook of positive organizational scholarship (pp. 125-137). Nova Iorque: Oxford University Press. doi: 10.1093/ oxfordhb/9780199734610.013.0010

Bruin, G. P, \& Henn, C. M. (2013). Dimensionality of the 9-item Utrecht Work Engagement Scale (UWES-9). Psychological Reports: Human Resources and Marketing, 112, 788-799. doi: 10.2466/01.03. PR0.112.3.788-799

Diener, E., Emmons, R. A., Larsen, R. J., \& Griffin, S. (1985). The satisfaction with life scale. Journal of Personality Assessment, 49, 71-75.doi: 10.1207/s15327752jpa4901_13

Diener, E., Wirtz, D., Tov, W., Kim-Prieto, C., Choi, D., Oishi, S., \& Robert Biswas-Diener, R. (2010). New well-being measures: short scales to assess flourishing and positive and negative feelings. Social Indicators Research, 97, 143-156. doi: 10.1007/s11205-009-9493-y 
Ferreira, M. C., Thadeu, S. H., Masagão, V., Gottardo, L., Gabardo, L., Souza, S. A., \& Mana, T. (2013). Escala de avaliações autorreferentes: características psicométricas em amostras brasileiras. Avaliação Psicológica, 12, 227-232. Recuperado de de http://pepsic.bvsalud.org/scielo.php?script=sci_arttext\&pid=S167704712013000200013\&lng=pt\&tlng=pt.

Fong, T. C.T., \& Ho, R. T. H. (2015). Dimensionality of the 9-item Utrecht Work Engagement Scale revisited: a Bayesian structural equation modeling approach. Journal of Occupational Health, 57(4): 353-8. doi: 10.1539/joh.15-0057-OA

Fong, T. C. T., \& Ng, S. M. (2012). Measuring engagement at work: validation of the Chinese version of the Utrecht Work Engagement Scale. International Journal of Behavioral Medicine, 19, 391-397. doi: 10.1007/s12529-011-9173-6

Gouveia, V. V., Barbosa, G. A., Andrade, E. O., \& Carneiro, M. B. (2005). Medindo a satisfação com a vida dos médicos no Brasil. Jornal Brasileiro de Psiquiatria, 54, 298-305.

Hayduk, L., Cummings, G., Boadu, K., Pazderka-Robinson, H., \& Boulianne, B. (2007). Testing! testing! one, two, three - Testing the theory in structural equation models! Personality and Individual Differences, 42, 841-850. doi:10.1016/j.paid.2006.10.001.

John, O.P., Donahue, E. M., \& Kentle, R. L. (1991). The Big Five InventoryVersions 4a and 54. Berkeley, CA: University of California, Berkeley, Institute of Personality and Social Research.

Judge, T. A., Erez, A., Bono, J. E., \& Thoresen, C. J. (2003). The core selfevaluations scale: development of a measure. Personnel Psychology, 56, 303-331. doi: 10.1111/j.1744-6570.2003.tb00152.x

Klassen, R. M., Aldhafri, S., Mansfield, C. F., Purwanto, E., Siu, A. F. Y., Marina W., ... Woods-McConney, A. (2012). Teachers' engagement at work: an international validation study. The Journal of Experimental Education, 80, 317-337. doi: 10.1037/a0026253

Littman-Ovadi, H., \& Balducci, C. (2013). Psychometric properties of the Hebrew version of the Utrecht Work Engagement Scale (UWES-9). European Journal of Psychological Assessment, 29, 58-63. doi: 10.1027/1015-5759/a000121

Mendonça, H., Caetano, A., Ferreira, M. C., Sousa, I. F., \& Silva, A. J. (2014). Florescimento no trabalho. In M. M. M. Siqueira (Org.), Novas medidas do comportamento organizacional: Ferramentas de diagnóstico e de gestão (pp. 172-177). Porto Alegre: Artmed.

Muthén, B. (1988). Some uses of structural equation modeling in validity studies: extending IRT to external variables. In H. Wainer \& H. Braun (Orgs.), Test validity (pp. 213-238). Hillsdale, NJ: Erlbaum.

Nerstad, C. G., Richardsen, A. M., \& Martinussen, M. (2010). Factorial validity of the Utrecht Work Engagement Scale (UWES) across occupational groups in Norway. Scandinavian Journal of Psychology, 51, 326-333. doi: 10.1111/j.1467-9450.2009.00770.x

Panthee, B., Shimazu, A., \& Kawakami, N. (2014). Validation of Nepalese version of Utrecht Work Engagement Scale. Journal of Occupational Health, 56, 421-429. doi: 10.1539/joh.14-0041-OA

Pereira, M. M., Ferreira, M. C., \& Valentini, F. (2016). Evidências de validade da escala de paixão pelo trabalho em amostras brasileiras. Manuscrito submetido para publicação.

Podsakoff, P. M., MacKenzie, S. B., \& Podsakoff, N. P. (2012). Sources of method biasing social science research and recommendations on how to control it. Annual Review of Psychology, 63, 539-69. doi: 10.1146/annurev-psych-120710-100452
Romero, E., Villar, P., Gómez-Fraguela, J. A., \& López-Romero, L. (2012). Measuring personality traits with ultra-short scales: a study of the Ten Item Personality Inventory (TIPI) in a Spanish sample. Personality and Individual Differences, 53(3), 289-293. doi:10.1016/j.paid.2012.03.035

Sass, D. A. (2011). Testing measurement invariance and comparing latent factor means within a confirmatory factor analysis framework. Journal of Psychoeducational Assessment, 29, 347-363. doi: $10.1177 / 0734282911406661$

Satorra, A., \& Bentler, P. M. (2001). A scaled difference chi-square test statistic for moment structure analysis. Psychometrika, 66, 507-514. doi:10.1007/BF02296192

Schaufeli, W.B. (2012). Work engagement. What do we know and where do we go? Romanian Journal of Applied Psychology, 14, 3-10. Recuperado de http://www.rjap.psihologietm.ro/Download/rjap141_1.pdf

Schaufeli, W. B. (2013). What is engagement? In C. Truss, K. Alfes, R. Delbridge, A. Shantz, \& E. Soane (Orgs.), Employee engagement in theory and practice. Londres: Routledge.

Schaufeli, W. B., \& Salanova, M. (2008). Enhancing work engagement through the management of human resources. In K. Näswall, M. Sverke, \& J. Hellgren (Orgs.), The individual in the changing working life (pp. 380-404). Cambridge: Cambridge University Press

Schaufeli, W. B., Bakker, A. B., \& Salanova, M. (2006). The measurement of work engagement with a short questionnaire: a cross-national study. Educational and Psychological Measurement, 66, 701-716. doi: 10.1177/0013164405282471

Schaufeli, W. B., Salanova, M., González-Romá. V., \& Bakker, A. B. (2002). The measurement of engagement and burnout: a confirmatory factor analytic approach. Journal of Happiness Studies, 3, 71-92. doi: 10.1023/A:1015630930326

Schweizer, K. (2011). Some thoughts concerning the recent shift from measures with many items to measures with few items. European Journal of Psychological Assessment, 27(2), 71-72. doi:10.1027/1015-5759/a000056

Seligman, M. E. P., \& Csikszentmihalyi, M. (2000). Positive psychology: an introduction. American Psychologist, 55, 5-14. doi: 10.1037//0003066X.55.1.5

Shimazu, A., Schaufeli, W. B., Kosugi, S., Suzuki, A., Nashiwa, H., Kato, A., ... Kitaoka-Higashiguchi, K. (2008). Work engagement in Japan: validation of the Japanese version of the Utrecht Work Engagement Scale. Applied Psychology: An International Review, 57, 510-523. doi: 10.1111/j.1464-0597.2008.00333.x

Silva, A. P. C., \& Ferreira, M. C. (2009). Escala de Satisfação Geral no Trabalho (Resumo). In Instituto Brasileiro de Avaliação Psicológica (Org.), Anais do IV Congresso Brasileiro de Avaliação Psicológica (p. 246). Campinas, SP: IBAP.

Siqueira, M. M. M. (2008). Satisfação no trabalho. In M. M. M. Siqueira (Org.), Medidas do comportamento organizacional: ferramentas de diagnóstico e gestão (pp. 265-274). Porto Alegre: Artmed.

Vallerand, R. J., \& Houlford, N. (2003). Passion at work: toward a new conceptualization. In S. W. Gilliland, D. D. Steiner, \& D. P. Skarlicki (Orgs.), Emerging perspectives on values in organization (pp. 175204). Greenwich, CT: Information Age Publishing.

Vazquez, A. C. S., Magnan, E. S., Pacico, J. C., Hutz, C. S., \& Schaufeli, W. B. (2015). Adaptation and validatio of the Brazilian Version of the Utrecht Work Engagement Scale. Psico-USF, 20, 207-217. doi: 10.1590/1413-82712015200202 
Vigoda-Gadot, E., Eldor, L., \& Schohat, L. M.(2012). Engage them to public service: Conceptualization and empirical examination of employee engagement in public administration. The American Review of Public Administration, 43, 518-538. doi: 10.1177/0275074012450943

Wefald, A. J., Mills, M. J., Smith, M. R., \& Downey, R. G. (2012). A comparison of three job engagement measures: examining their factorial and criterion-related validity. Applied Psychology: Health and Well-Being, 4, 67-90. doi: 10.1111/j.1758-0854.2011.01059
Williams, L. J., \& Anderson, S. E. (1991). Job satisfaction and organizational commitment as predictors of organizational citizenship and in-role behaviours. Journal of Management, 78, 374-381.doi: 10.1177/014920639101700305

Zecca, G., Györkös, C., J. Becker, J., Massoudi, K., Bruin, G. P., \& Rossier, J. (2015). Validation of the French Utrecht Work Engagement Scale and its relationship with personality traits and impulsivity. Revue Européenne de Psychologie Appliquée, 65, 19-28. doi: 10.1016/j. erap.2014.10.003

Maria Cristina Ferreira, Doutora em Psicologia pela Fundação Getúlio Vargas (RJ), é Professora Titular e Coordenadora do Programa de Pós-Graduação em Psicologia da Universidade Salgado de Oliveira (UNIVERSO). Endereço para correspondência:

Rua Marquês de Valença, 80 Apto. 602 - Tijuca, Rio de Janeiro - RJ CEP 20550-030. Telefone: (21) 98898-9207. E-mail: mcris@centroin.com.br

Felipe Valentini, Doutor em Psicologia pela Universidade de Brasília (UnB) e Pós-Doutorado pela Universidade Salgado de Oliveira (UNIVERSO), é Professor Titular do Programa de Pós-graduação em Psicologia da Universidade Salgado de Oliveira (UNIVERSO). E-mail: valentini.felipe@gmail.com

Bruno Figueiredo Damásio, Doutor em Psicologia pela Universidade Federal do Rio Grande do Sul (UFRGS), é Professor Adjunto da Universidade

Federal do Rio de Janeiro (UFRJ). E-mail: bf.damasio@gmail.com

Luciana Mourão, Doutora em Psicologia pela Universidade de Brasília (UnB)

e Estágio pós-doutoral realizado no Instituto Universitário de Lisboa, é

Professora Titular do Programa de Pós-graduação em Psicologia da Universidade Salgado de Oliveira (UNIVERSO). E-mail: mourao.luciana@gmail.com

Juliana Barreiros Porto, Doutora em Psicologia pela Universidade de Brasília (UnB), é Professora adjunto na Universidade de Brasília (UnB). E-mail: porto.juliana@gmail.com

Renata Silva de Carvalho Chinelato, Doutora em Psicologia pela Universidade Salgado de Oliveira (UNIVERSO), é Professora Assistente no Centro Universitário Estácio Juiz de Fora (CENUNESJF). E-mail: resilvajf@gmail.com

Vladimir Pinto Novaes, Mestrado em Psicologia pela Universidade Salgado de Oliveira (UNIVERSO), é Doutorando da Universidade Salgado de Oliveira (UNIVERSO). E-mail: vladimir_novaes@hotmail.com

Michelle Morelo Pereira, Mestrado em Psicologia pela Universidade Salgado de Oliveira (UNIVERSO), é Professora Substituta na Universidade Federal Fluminense (UFF) e Doutoranda da Universidade Salgado de Oliveira (UNIVERSO).E-mail: morelo.mi@gmail.com

Recebido em 05.Nov.15

Revisado em 13.Mai.16

Aceito em 13.Set.16 